\title{
Integration platform for optical switching of magnetic elements
}

Sobolewska, Elżbieta, Pelloux-Prayer, Johan, Becker, Hanna, Li, Guanqiao, Davies, Carl, et al.

Elżbieta K. Sobolewska, Johan Pelloux-Prayer, Hanna Becker, Guanqiao Li, Carl S. Davies, Clemens J. Krückel, Luis Avilés Félix, Aurélien Olivier, Ricardo Sousa, Ioan-Lucian Prejbeanu, Andrei I. Kiriliouk, Dries Van Thourhout, Theo Rasing, Farshad Moradi, Martijn J. R. Heck, "Integration platform for optical switching of magnetic elements," Proc. SPIE 11461, Active Photonic Platforms XII, 114612B (31 August 2020); doi: 10.1117/12.2567662

SPIE. Event: SPIE Nanoscience + Engineering, 2020, Online Only 


\title{
Integration platform for optical switching of magnetic elements
}

\author{
E. K. Sobolewska ${ }^{a}$, J. Pelloux-Prayer ${ }^{a}$, H. Becker ${ }^{a}$, G. Li ${ }^{b}$, C. S. Davies ${ }^{\text {b, }}$ \\ C. J. Krüickel ${ }^{\mathrm{d}}$, L. Avilés-Félix ${ }^{\mathrm{e}}$, A. Oliviere , R. C. Sousa ${ }^{\mathrm{e}}$, I. L. Prejbeanu ${ }^{\mathrm{e}}$, A. I. Kirilyuk ${ }^{\mathrm{b}, \mathrm{c}}$, \\ D. Van Thourhout ${ }^{\mathrm{d}}$, T. Rasing ${ }^{\mathrm{b}}$, F. Moradi ${ }^{\mathrm{a}}$ and M. J. R. Heck ${ }^{\mathrm{a}^{*}}$ \\ ${ }^{a}$ Department of Engineering, Aarhus University, Finlandsgade 22, 8200 Aarhus, Denmark; \\ b-Institute for Molecules and Materials, Radboud University, Heyendaalseweg 135, 6525 AJ \\ Nijmegen, The Netherlands; \\ ${ }^{c}$ FELIX Laboratory, Radboud University, Toernooiveld 7, 6525 ED Nijmegen, The \\ Netherlands; \\ d Photonics Research Group, Department of Information Technology, Ghent University - \\ IMEC Ghent, 9052 Ghent, Belgium; \\ e Spintec, Université Grenoble Alpes, CNRS, CEA, Grenoble INP, IRIG-SPINTEC, \\ 38000 Grenoble, France;
}

\begin{abstract}
We present a detailed investigation of a novel platform for integration of spintronic memory elements and a photonic network, for future ultrafast and energy-efficient memory. We designed and fabricated magnetic tunnel junction (MTJ) structures based on (Tb/Co) $\times 5$ multilayer stack with optically switchable magnetization. Optical single-pulse measurements allowed us to estimate the value of the stray field present in the parallel configuration, which prevents the structure from all-optical switching. We performed numerical calculations based on the Finite Difference Time Domain method and ellipsometry measurements of $(\mathrm{Tb} / \mathrm{Co})_{x 5}$ to compute the absorption by the MTJ structure. Simulation results are in good agreement with the experimental measurements, where we implemented a thermal model to estimate effective absorption in the pillar. These estimations showed up to $14 \%$ absorption of the incident optical power in 300-nm-wide MTJ. Moreover, we designed and realized an integrated optical network with focusing structures to efficiently guide and couple the light into the MTJs. We show a chain of necessary steps to obtain the threshold value of the switching energy, and our results presenting a path forward for full system integration of optically switchable MRAM technology.
\end{abstract}

Keywords: integrated photonics, photonic, spintronic, terbium, cobalt, all-optical switching, magnetic memory

*m.heck@tue.nl

\section{INTRODUCTION}

Nowadays, when working digitally is not an option but rather the requirement, there is a strong need for the development of more efficient data storage elements with higher speed and less power consumption. Therefore, for the past decade, it has been an interdisciplinary effort to improve the magnetization-switching process used as a fundamental phenomenon in the magnetic memory device.

Active Photonic Platforms XII, edited by Ganapathi S. Subramania, Stavroula Foteinopoulou, Proc. of SPIE Vol. 11461, 114612B · (C) 2020 SPIE · CCC code: 0277-786X/20/\$21 · doi: 10.1117/12.2567662 
Magnetoresistive random-access memory (MRAM) is one promising technology, offering high read and write speed (times of $10 \mathrm{~ns} / \mathrm{b}$ ) and energy-efficient performance thanks to its non-volatility and low voltage operation $(<0.3 \mathrm{~V})$ [1][2]. Additionally, MRAM technology offers practically unlimited endurance [3] and its fundamental building block, the magnetic tunnel junctions (MTJs), allows for the integration of novel materials. The all-optical magnetization reversal of magnetic layers in the MTJs with picosecond optical pulses [4] is of particular interest as it shows the potential for energyefficient and faster magnetoresistive random access memory (MRAM) [5]. This phenomenon relies on ultrafast thermal effects known as all-optical switching (AOS) of the magnetization. AOS was seen in certain multilayer and alloy materials [6][7]. Rare-earth (RE) - transition metal (TM) attracts an increasing amount of interest, mainly due to their potential in the field of magneto-optical recording [8][9]. Their integration into the MTJ layer stack enabled the vision towards optically written MRAM elements [10], which can be read electronically. This approach requires memory elements that are optically and electronically accessible, which can be achieved by utilizing transparent conductive oxides, like indium tin oxide (ITO) [11].

In addition, a future memory technology should also show the potential for an integrated and scalable path forward. It requires an integrated photonic switching network that can deliver and a couple of short optical pulses to the MTJ elements, at a low optical loss and with low energy consumption. The SPICE [12] project targets the integration of optically accessible MRAM technology with integrated photonics. This hybrid integration approach for photonic-spintronic integration could have the added advantage of opening up optical bandwidths and low-energy data transmission to data storage and electronics in general. The silicon photonics platform was initially identified as the most suitable platform for this hybrid integration approach, offering components with comparatively small footprints and thus dense photonic integration, and energy-efficient electro-optic modulation, enabling integrated optical pulse routing networks.

The following presents an overview of our efforts to combine modern MRAM technology with a photonics platform. We describe critical steps towards finding the energy threshold values for efficient AOS. We characterize MTJ layers in terms of their optical properties and investigate their role in the switching mechanism. We present components of the photonic platform, which can provide focused and high energy light into the MTJ devices, which show potential for future large-scale integration.

\section{MATERIALS AND METHODS}

After the optical observation of all-optical single pulse switching of the Tb/Co half electrode [8][9], we fabricated the MTJ electrode [10]. The device consists of the MTJ stack shown in Figure 1(a) and is topped with a $150 \mathrm{~nm}$ ITO layer. The Tb/Co bilayer was repeated five times in the MTJ stack and is magnetically coupled to the top $\mathrm{CoFeB}$ layer. The deposition process of all layers was optimized to achieve the best performance of the magnetic tunnel junction elements [8] [11].

This following section introduces the methods and material characterization of the material layer stack we developed for optically switchable MTJs. These MTJs are built with an optically switchable 'storage' layer, while the 'sensing' layer is not susceptible to change by short optical pulses. The performance of the devices was established by measuring the resistance dispersion between the low and high resistance states of the MTJs. The typical high resistance state $\left(\mathrm{R}_{\mathrm{AP}}\right)$ corresponds to the 'free' layer's (Tb/Co multilayers) magnetization in the anti-parallel state to the 'reference' layer $(\mathrm{CoFeB}$ below $\mathrm{MgO}$ ) and the low resistance state $\left(\mathrm{R}_{\mathrm{P}}\right)$ is obtained for the parallel state (Figure 1(c)) [8][13]. 


\subsection{Structure of the multilayers stack}

The device consists of the MTJ stack shown in Figure 1(a) and is topped with a $150 \mathrm{~nm}$ ITO layer. ITO layer as a top electrode ensured optical access to the AOS layer and electrical contact within this stack. The magnetization state of the device can be controlled by an external magnetic field, leading to hysteresis as shown in Figure 1c, indicating the high resistance state $\left(\mathrm{R}_{\mathrm{AP}}\right)$ and low resistance state $\left(R_{P}\right)$. This allows us to measure the resistance dispersion over all fabricated devices as well as their tunnel magnetoresistance (TMR) value, defined by:

$$
T M R=\frac{R_{A P}-R_{P}}{R_{P}}
$$

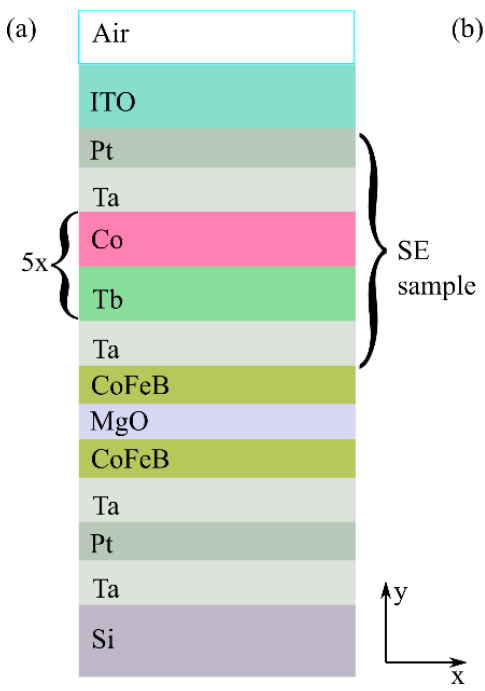

(b) EM Plane wave $(0.8-1.8 \mu \mathrm{m})$
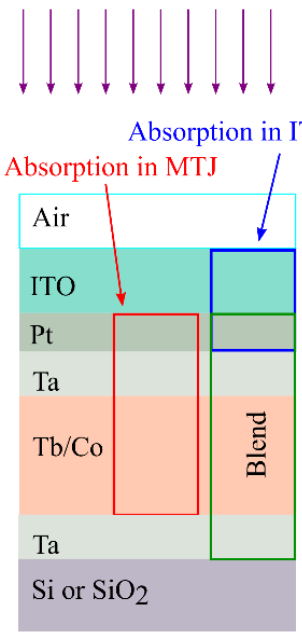

(c)

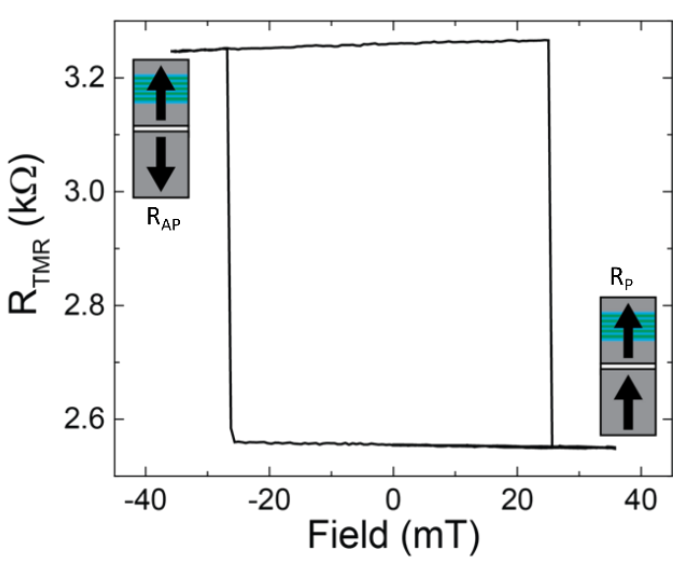

Figure 1 Schematic representation of (a) The multilayer structure of the full device; (b) Simplified structure of the stack for ellipsometry measurements and FDTD calculations of reflection, transmission and absorption. (c) The magnetic field hysteresis of MTJ device, with $T M R$ resistance as a function of the applied magnetic field.

Knowledge about the optical properties of the materials in the multilayer stack is crucial for estimating the switching power and absorbed energy. Most of the materials used in the MTJ pillar are well known. However, there were not many references for the most essential of AOS materials like terbium (Tb) and cobalt (Co). For that purpose, we characterized the multilayer samples with spectroscopic ellipsometry in as-deposited state and after each of the annealing steps. Full sheet film samples were annealed in vacuum for $10 \mathrm{~min}$ at $250^{\circ} \mathrm{C}$ and subsequently again for $10 \mathrm{~min}$ at $300^{\circ} \mathrm{C}$ in a hightemperature furnace.

Ellipsometry measurements were done with a Sentech SE 850 PV spectroscopic ellipsometry (SE) in a range of optical wavelengths from $0.7 \mu \mathrm{m}$ to $1.8 \mu \mathrm{m}$ and at incidence angles of $60^{\circ}, 65^{\circ}$ and $70^{\circ}$. 
From SE measurements, we obtained a set of the amplitudes ratio $(\psi)$ and phase shift $(\Delta)$ for both polarisation $\left(R_{p}, R_{s}\right)$ of the reflected light which is described by the relation [14][15]:

$$
\frac{R_{p}}{R_{S}}=\tan (\psi) e^{i \Delta}
$$

The Kramers-Kronig regression model for the ellipsometry parameters $\Delta$ and $\psi$ determined the optical constants of the measured thin films [16]. To analyze obtained data, we constructed three optical models taking into account the silicon $(\mathrm{Si})$ substrate and other layers present in the sample.

We compared results from three different analytical models: "multilayers", "single-layer" and "blend". The thicknesses of all layers were fixed during the entire analysis. For Pt [17], Cu [18] and Ta [17], we used known optical constants and left only the $\mathrm{Tb} / \mathrm{Co}$ as a fitting parameter. We additionally used the optical constant of Co [19] in the "multilayer model" that replicated the original structure of the stack with 5 alternately repeated layers of $\mathrm{Tb}$ and $\mathrm{Co}$. This model was not further investigated since we achieved the highest value of the mean square error - MSE [15], which describes the quality of the fit, comparing all three models. Such a high value of the MSE suggest an insufficient number of data for Co in the near-infrared red (NIR) region (only 5 points) or required more precise input parameters. The second - "single-layer" model assumed all repeated layers of $\mathrm{Tb}$ and $\mathrm{Co}$ as one layer with a total thickness of the sum of their thicknesses. The third approach, called "blend" used an effective medium approximation to calculate the complex refractive index of all the thin films in the stack as one layer. For the estimation of the dielectric function, the analysis was based on a parametric model using the Drude-Lorentz oscillator model for $\mathrm{Tb}$ and $\mathrm{Tb} / \mathrm{Co}$ [19] thin films and a series of layers with known optical parameters (for $\mathrm{Pt}, \mathrm{Ta}, \mathrm{Cu}, \mathrm{Si}[19]$ ). Thicknesses of all layers were controlled during the optimization of the fabrication process, and for the SE measurements, they were used as fixed input parameters throughout the fitting process. The top Pt-layer was assumed as a perfect thin film with a neglectable surface roughness of RMS $<0.5 \mathrm{~nm}$. We used those analytical models for samples with different $\mathrm{Tb}$ to Co ratios (1.1:1.5 nm; 1.4:1.5 $\mathrm{nm}$ and 1.3:1.5 nm) and made further adjustments due to changes in the structure caused by the annealing process.

For estimation of the absorbed optical power by the MTJ multilayers stack, we used the commercially available Finite Difference Time Domain (FDTD) simulation tool from Lumerical Solution Inc. [22]. We established a 2D numerical model with a maximum mesh ("Yee-cell") size of $1 \mathrm{~nm}$ in both lateral and vertical dimensions. To mimic the structure of the sample, the simulation cell contained a multilayers stack of $\operatorname{Air} / \mathrm{ITO}_{(150 \mathrm{~nm})} / \mathrm{Pt}_{(2 \mathrm{~nm})} / \mathrm{Ta}(2 \mathrm{~nm}) /[\mathrm{Tb} / \mathrm{Co}]_{(13 \mathrm{~nm})} / \mathrm{CoFeB}_{(2 \mathrm{~nm})} / \mathrm{MgO}_{(3}$ $\mathrm{nm}) / \mathrm{Si}(5000 \mathrm{~nm})$. Due to computation requirements and simulation time, the second layer of Ta $(0.2 \mathrm{~nm})$ and all metallic layers below $\mathrm{MgO}$ was not included in the model (Figure 1(b)). The small size of the pillar $(\max .0 .3 \mu \mathrm{m})$ comparing to the measured spot size of the light $(2.5 \mu \mathrm{m}$ [23]) allowed implementing the electromagnetic (EM) plane wave as a light source in the $0.6-1.6 \mu \mathrm{m}$ wavelengths range with polarisation parallel to the $\mathrm{X}$-axis. The size of the simulation region was set to the size of the largest MTJ $(0.3 \mu \mathrm{m})$. To suppress unwanted reflections and mimic the infinite thicknesses of the substrate and air layer, a perfect matching layer (PML) and Bloch boundary conditions were set in $x$ and $y$ direction, respectively. To accurately reproduce the experimental conditions, we applied a complex refractive index for ITO and $\mathrm{Tb} / \mathrm{Co}$ based on our spectroscopic ellipsometry measurements. We interpolated the permittivity of $\mathrm{Pt}, \mathrm{Ta}, \mathrm{CoFeB}$ [23], $\mathrm{MgO}$ [25] and Si to fit into our FDTD model. We compared our reflection and transmission results with analytical calculations based 
on the analytical transfer matrix method [15][26]. This comparison allowed us to confirm the correctness of the model and calculate absorption profile $\left(\mathrm{P}_{\mathrm{abs}}\right)$ of $\mathrm{Tb} / \mathrm{Co}$, ITO and MTJ layers using the relation [27]:

$$
P_{a b s}=-0.5 \omega|E|^{2} \operatorname{Im}(\varepsilon) .
$$

Where $\omega$ is frequency, $E$ electric field intensity and $\varepsilon$ is an imaginary part of the permittivity.

\subsection{Thermal model}

All-optical switching effects were mainly characterized in terms of optical fluence required to trigger it in a full sheet film sample. These values cannot simply be applied to patterned structures below the diffraction limit [28][29], due to the modification of the absorption of the laser pulse by the size and shape of the structure. The effective absorption of the laser pulse must be evaluated to obtain AOS threshold values for a patterned structure [29].

For that purpose, we applied a lumped elements model as a thermal equivalent model for the MTJ, where we considered our MTJ as a thermal element with a mean thermal resistance and a mean heat capacitance. We computed these values for the full MTJ structure with literature values of thermal conductivity for each of the layers [Table 1 [30]]. The thermal model was built on the basic principle of heat conduction in a material slab [31][32][33]:

$$
\frac{\mathrm{Q}}{\mathrm{t}}=\kappa \mathrm{A} \frac{\left(\mathrm{T}_{\text {hot }}-\mathrm{T}_{\text {cold }}\right)}{d}
$$

Where $\mathrm{Q}$ is the heat transferred in the time $\mathrm{t}$, and where $\kappa$ is the thermal conductivity of the slab. A is the surface area normal to the heat flux, $\mathrm{T}_{\text {hot }}$ and $\mathrm{T}_{\text {cold }}$ are the temperatures on the two sides of the slab, and $d$ is the thickness of the slab. The thermal resistance $\left(R_{t h}\right)$ of each layer was taken as $d / \kappa$. Thus equation (4) can be rearranged to

$$
\frac{\mathrm{Q}}{\mathrm{At}}=\frac{\left(\mathrm{T}_{\mathrm{hot}}-\mathrm{T}_{\mathrm{cold}}\right)}{R_{t h}}
$$

This approach assumed measuring the increase of the temperature in the MTJ due to laser heating at equilibrium, meaning that the heat flux is constant: heat ${ }_{\text {in }}=$ heat $_{\text {out. }}$ This allowed us to ignore the thermal capacitance of the materials and to consider each part of the junction as thermal resistors connected in an equivalent circuit [31]. Moreover, we isolated the bottom and top contact of the structure because we considered that measured thermal change of resistance was mainly due to the resistivity change of the MTJ structure and not the contacts. In our equivalent circuit, we separated the entire MTJ layer stack into three elements (Figure 2): the two contacts and the MTJ, with the latter consisting of 'storage' layer, tunnelling layer $(\mathrm{MgO})$, and the 'sensing' layer. We considered that the laser illumination results in a heat source qheat located between the MTJ and the top contact. We placed the MTJ ( $\left.\mathrm{R}_{\text {th,MTJ }}\right)$ and the bottom contact $\left(\mathrm{R}_{\mathrm{th}, \mathrm{bot}}\right)$ in series and the top contact $\left(\mathrm{R}_{\mathrm{th}, \mathrm{ITO}}\right)$ in parallel, as shown in Figure 2. The parallel resistance was due to the boundary condition of our system: we have the top of the ITO in contact with air and thus considered at room temperature. The bottom contact 
was in thermal contact with the Si bulk substrate, and we considered the bulk to be at room temperature given its size relative to the MTJ and the thermal conductivity of Si (Table 1). This gave us two antiparallel heat flows from the heat source to the top contact and from the heat source to the bottom contact. The heat source location inside our circuit resulted in an energy minimum, which was identified by splitting the MTJ resistance in two fractional resistances $x R_{\text {thMTJ }}$ and (1-x) $R_{\text {thMTJ }}$ and solving the resulting equivalent circuit:

$$
R_{t h, e q}(x)=\frac{\left((1-x) R_{t h, M T J}+R_{t h, b o t}\right)\left(R_{t h, t o p}+(x) R_{t h, M T J}\right)}{\left(R_{t h, M T J}+R_{t h, b o t}\right)+R_{t h, t o p}}
$$

A more precise analysis of this system would require a full temporal simulation of both the laser absorption and the thermal response of the structure. For simplicity, the contact and substrate were taken as cylinders of the same diameter as the MTJ during the calculation.

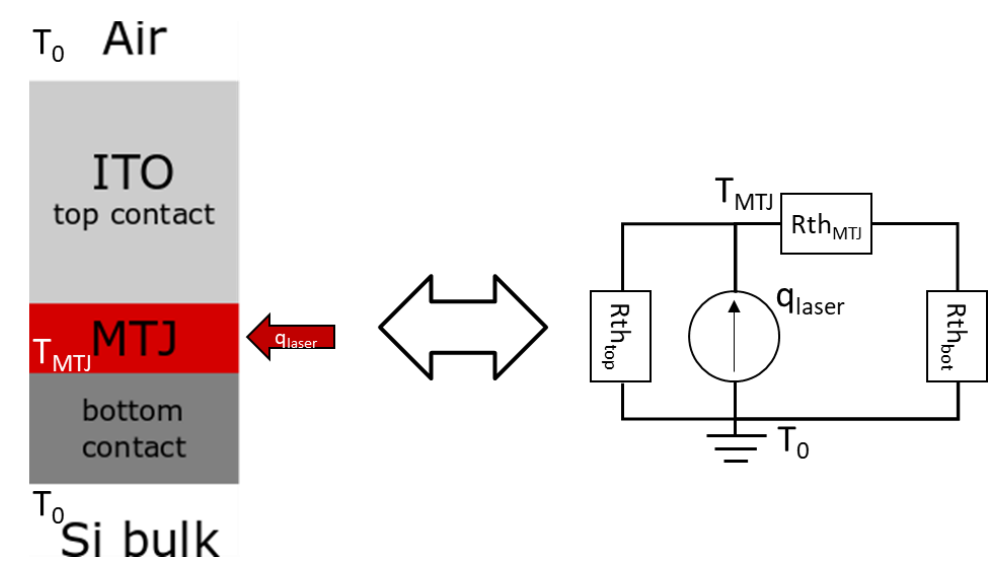

Figure 2 Equivalent circuit of the thermal model of heat flow in an MTJ during laser irradiation (qlaser).

We can express the equivalent thermal resistance for our equivalent circuit as follows:

$$
R_{t h, e q}=\frac{\left(R_{t h, M T J}+R_{t h, b o t}\right) R_{t h, t o p}}{\left(R_{t h, M T J}+R_{t h, b o t}\right)+R_{t h, t o p}}
$$

This leads to the equation of the heat flow (qheat) through the MTJ in equilibrium:

$$
q_{\text {heat }}=\frac{\mathrm{Q}}{\mathrm{A}_{\mathrm{MTJ}} \cdot \mathrm{t}}=\frac{\left(\mathrm{T}_{\mathrm{MTJ}}-\mathrm{T}_{0}\right)}{R_{t h, e q}}=\frac{\mathrm{T}_{\mathrm{meas}}}{R_{t h, e q}},
$$

where both $\mathrm{T}_{0}$ (room temperature), and $\mathrm{T}_{\text {meas }}\left(\mathrm{T}_{\mathrm{MTJ}}-\mathrm{T}_{0}\right)$ come from the performed experiments.

It was possible to estimate the coupling efficiency $\alpha_{\text {eff }}$ of the MTJs, by comparing the qheat value to the measured out-coupled laser power $\left(\mathrm{P}_{\text {laser }}\right)$ :

$$
\alpha_{\mathrm{eff}}=\frac{q_{\text {heat }} \cdot A_{M T J}}{P_{\text {laser }}}
$$


Table 1 Thermal resistances and heat capacitances for materials used in the equivalent model [30].

\begin{tabular}{|l|l|l|}
\hline & $\mathrm{R}_{\mathrm{th}}\left(10^{-9} \mathrm{~m}^{2} \mathrm{~K} / \mathrm{W}\right)$ & $\mathrm{C}_{\mathrm{th}}\left(10^{-15} \mathrm{~J} / \mathrm{K} \mathrm{m}^{2}\right)$ \\
\hline MTJ & 1.50 & 2.55 \\
\hline Pt bottom contact & 1.43 & 0.53 \\
\hline ITO top contact & 14.7 & 28.2 \\
\hline Si bulk & 0.417 & 0.0059 \\
\hline
\end{tabular}

\subsection{Single-pulse switching measurement}

Optical switching of the MTJ with the shown layer stack from Figure 1(a) was experimentally investigated with a free-space optical setup, allowing placement of the MTJs in an external magnetic field. The coercive field of the Tb/Co bilayer lies typically above $100 \mathrm{mT}$, which was outside of our experimental capacities [8]. However, the bottom CoFeB layer has a typical coercive field below 30 mT (Figure 1(c)). This allowed us to measure the tunnelling magnetoresistance as a function of the applied magnetic field where the magnetization of the bottom $\mathrm{CoFeB}$ layer was changed with respect to the magnetization of the top $\mathrm{CoFeB}$ layer, which was coupled to $\mathrm{Tb} / \mathrm{Co}$. In the case where the magnetic top and bottom layers were parallel with respect to each other, we observed a relatively low resistance while in the case of an anti-parallel configuration a relatively high resistance. We performed experiments with two different initial states of the magnetization where we excited the MTJ device with a laser pulse with a pulse duration of $2 \mathrm{ps}$ and a central wavelength of $800 \mathrm{~nm}$ at a fluence of 20 $\mathrm{mJ} \mathrm{cm}-2$. After each pulse, we measured the voltage of the device as a function of the applied magnetic field using a Keithley 2700 multimeter under a DC current of $5 \mu \mathrm{A}$ which was provided by a Keithley 2400 source meter.

\subsection{Grating couplers}

Successful photonic-spintronic integration requires layer couplers, which are able to vertically couple optical signals up/down to the spintronic elements, depending on the integration strategy. The silicon photonics platform offers a high-refractive-index contrast and thus enables compact device design and high photonic integration densities. Diffractive grating couplers are the standard method for coupling optical signals into the silicon photonic chip [34] and have the potential for highly efficient coupling [35].

Compact out-of-plane focusing grating couplers (FGC) was designed with footprints of around $5 \times 5 \mu \mathrm{m}^{2}$ for the $1 \mathrm{D}$ components and around $10 \times 10 \mu \mathrm{m}^{2}$ for the 2D FGCs. Device areas in this range allow for both: optical-bandwidths exceeding the 100nm simulation window and diffraction-limited focusing to micron-/submicron sized focal spot sizes [23]. The design principle used the phasematching condition to ensure constructive interference in the focal spot located just a few micrometres above the device plane. The resulting curved and chirped 1D/2D gratings were optimized based on a coupled simulation routine using MATLAB and a full 3D FDTD simulator [22] with promising results published in [23]. 


\section{RESULTS AND DISCUSSION}

\subsection{Optical properties of MTJ's layers}

The results of the spectroscopic ellipsometry used the multilayer structure presented in Figure 1(a). Due to a strong dependence of transparent conductive oxide's (TCO) optical properties on deposition conditions, we used samples without the ITO electrode, which was measured separately. Additionally, the first capping layer of $\mathrm{Ta}$ was substituted for $\mathrm{Cu}$ layer as presented on the first version of AOSMTJ [1]. The complete investigated multilayers stack was built with $\mathrm{Si} / \mathrm{Ta}(30 \AA) /\left[\mathrm{Co}(15) / \mathrm{Tb}\left(\mathrm{t}_{\mathrm{Tb}}\right)\right] \times 5 / \mathrm{Cu}(20 \AA) / \mathrm{Pt}(30 \AA)$, where the thickness of the $\mathrm{Tb}\left(\mathrm{t}_{\mathrm{Tb}}\right)$ varied from 1.1 to $1.5 \mathrm{~nm}$.

Introducing the $\mathrm{Tb} / \mathrm{Co}$ as a "single-layer" with a thickness of the sum of the $\mathrm{Tb} / \mathrm{Co}$ layers showed a significantly higher quality of the fitting procedure (MSE $<1$ ), which suggested correctness of the analytical model. Results for the best fit are presented in Figure 3. Samples with all investigated Tb's thicknesses showed very high values of the Tb/Co's complex refractive index (ñ) (Figure 3), which agreed with a primary assumption based on results presented by Palik et al. of Tb [37] and Co [38] separately. Annealing of these samples at $250^{\circ} \mathrm{C}$ changed the results insignificantly. Such behaviour is characteristic of multilayers stacks exhibiting a "crystalline-like structure" [39]: The repeated layers with nanometer-scale thicknesses create an arrangement similar to the crystal's lattice. Such a structure is more resilient to temperature as well as shows higher values of $\mathrm{n}$ and $\mathrm{k}$ than in the amorphous form with same materials composition [40]. Substantial increase of the fitting quality also suggested improvement of the layers' morphology.

Additional annealing at $300^{\circ} \mathrm{C}$ followed by the ellipsometry measurement with the same analytical model as before showed significant changes of $\mathrm{Tb} / \mathrm{Co}$ layer's complex refractive index. We observed modifications of the real part of the refractive index $(\operatorname{Re}(\tilde{\mathrm{n}}))$ in two wavelengths regions. For wavelengths below $\sim 1 \mu \mathrm{m}$, the $\operatorname{Re}(\tilde{\mathrm{n}})$ increased by $\sim 40 \%$ compared to the as-deposited sample, were for the region above $1.3 \mu \mathrm{m} \operatorname{Re}(\tilde{\mathrm{n}})$ decreased by $\sim 30 \%$. These changes in the structure's morphology were results of the annealing in the high temperature. On the other hand, the extinction coefficient $(\operatorname{Im}(\tilde{\mathrm{n}}))$ showed a significant decrease in all the regions around $1.2 \mu \mathrm{m}$. Reduction of $\sim 89 \%$ of $\operatorname{Im}(\tilde{\mathrm{n}})$ at $1.55 \mu \mathrm{m}$ relates directly to a decrease of the absorbed optical power, which could explain the observed lack of AOS in MTJs after annealing at $300^{\circ} \mathrm{C}$ [8]. Even though the quality of the fit was at an acceptable level, it was much higher than before annealing for the same model and sample (MSE 10 [41]).

The third analytical model (based on effective medium approximation) consists of only 3 materials; air, "blend layer" (all the layers between ITO and Si) and Silicon substrate (Figure 3(c)). Here, the MSE decreased to below 1 for the sample annealed at $250^{\circ} \mathrm{C}$, and below 2 for the further annealing at $300^{\circ} \mathrm{C}$, which suggested a high quality of the fitting process. Annealing procedure is usually performed to improve the crystallinity of the structure and their optical properties [42][43][44]. However, in most literature cases, the investigated materials were oxides and semiconductors. In our case, the decrease of the refractive index happened above a particular temperature. During the annealing, the morphology and interfaces between layers underwent changes leading to interfaces with more indistinction between layers, which influence the "crystalline-like structure". Performing the annealing at high temperature with an access of air can introduce a generation of oxides with some of the metallic layers. Presence of the oxides in non-crystalline form 
would decrease the optical parameters of the stack [45][46]. Lower complex refractive index of "the blend" in comparison to the previous method is understandable, due to the presence of other metals in the stack. Especially $\mathrm{Cu}$, Ta and Pt show $\operatorname{Re}(\tilde{\mathrm{n}})$ with values in the range of $1-2$ in the NIR range. The substantial decrease of the extinction coefficient in the region above $1.4 \mu \mathrm{m}$ suggested a significant influence of the Tb/Co multilayers in the entire MTJ.
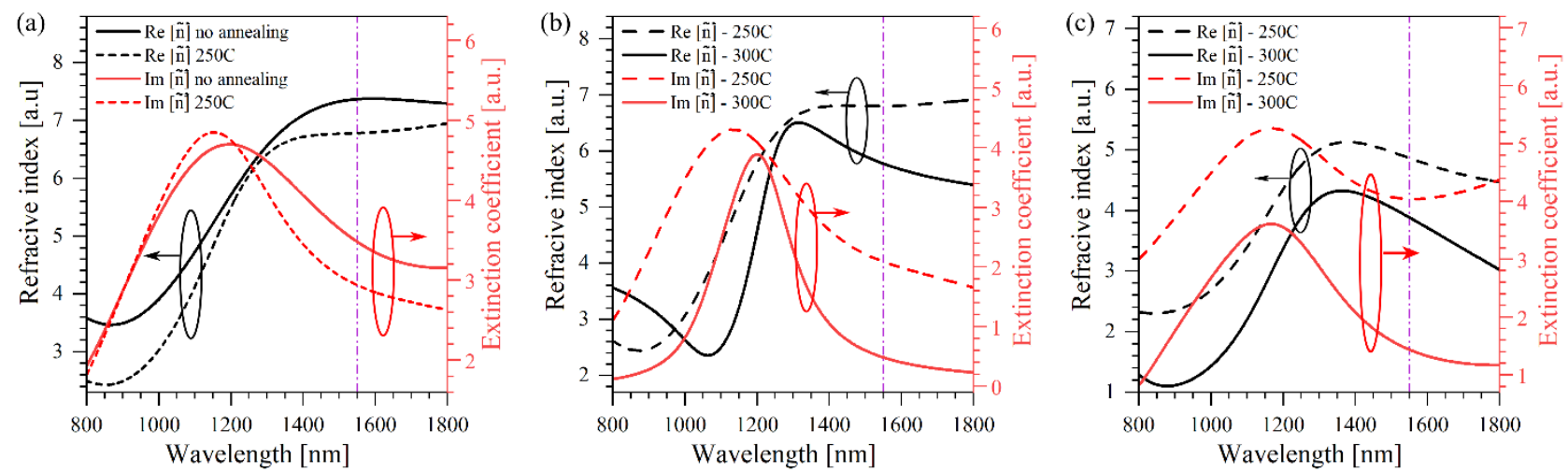

Figure 3 Near-infrared refractive index Re[ñ], and extinction coefficient $\operatorname{Im}[\tilde{n}]$ of $T b_{1.4} / C_{1.5}$ structure based on the spectroscopic ellipsometry results with single-layer model: (a) before and after annealing at $250^{\circ} \mathrm{C}$, (b) the sample with $\mathrm{Tb}_{1.1} / \mathrm{Co}_{1.5}$ annealed at $250^{\circ} \mathrm{C}$ and $300^{\circ} \mathrm{C}$. (c) The complex refractive index of the "blend" $\left(\mathrm{Pt} / \mathrm{Cu} /\left(\mathrm{Tb}_{1.1} / \mathrm{CO}_{1.5}\right) / \mathrm{Ta}\right)$ annealed at $250^{\circ} \mathrm{C}$ and subsequently at $300^{\circ} \mathrm{C}$.

No negligible optical losses in each layer of the stack require taking into account all the materials present in the pillar for estimating the absorption of the MTJ. Based on obtained results from the ellipsometry measurements, we build a numerical model of the multilayers stack to estimate the optical energy that is absorbed by the elements responsible for AOS. In our model, the absorption of the MTJ included all the metallic layers between ITO and CoFeB (Figure 1(b)). Preliminary simulation's results of ITO showed that the type of underlying material and substrate had a crucial influence for the reflection (R), transmission (T) and absorption (A) of the sample Numerical results were compared with experimental one [11] and showed a similar trend of R, T, A curves. We observed a discrepancy of $\sim 15 \%$ transmission in favour of calculations, which is an expected occurrence, due to the perfect conditions the simulation model assumes as well as the possible differences of optical parameters of the substrate and $\mathrm{Pt}$ thin film. Changing the substrate from $\mathrm{SiO}_{2}$ to $\mathrm{Si}$ resulted in an increase of the reflection at $1550 \mathrm{~nm}$ wavelength from $\sim 11 \%$ to $31 \%$ and the decrease of the transmission from $\sim 67 \%$ to $52 \%$. Those changes are natural consequences of a higher difference in refractive index between substrate and ITO.

After implementing additional materials presented in the stack, we performed a series of FDTD calculations to obtain the absorptions of each layer (Figure 4(a)). In our model, the "ITO layer" contained ITO and Pt, and MTJ layer covered Pt, Ta and (Tb/Co) thin films. Due to additional metals in the stack, the reflection increased from $31 \%($ ITO + Pt) to $48 \%$ at $1550 \mathrm{~nm}$ wavelength. Based on this model, we calculated that the MTJ layer absorbed around $17.5 \%$ of the optical power at $1550 \mathrm{~nm}$, with a maximum peak of $\sim 32 \%$ at $1050 \mathrm{~nm}$. We noticed that a similar percentage of the energy was lost due to absorption in ITO ( 16.9\% at $1550 \mathrm{~nm}$, with a peak of $\sim 24.5 \%$ at $1088 \mathrm{~nm})$. Absorption in the junction can be increased by implementing antireflection coating or fabricating an additional structure that can enhance the electromagnetic field around and inside the pillar. 


\subsection{Coupling efficiency based on the thermal model}

We aimed to characterize AOS events in nanometre-scaled MTJ pillars, for which optical absorption values are critical parameters in estimating the optical power required to switch the AOS-layer. Here, we described the estimation of the optical absorption in MTJ pillars of 200nm and 300nm diameter using laser light at a wavelength of $1550 \mathrm{~nm}$. By measuring the thermal dependence of the MTJ's resistive high state and low state, we could calculate the effective absorption of the laser light by the pillar.

We calibrated the electrical characteristic of measured MTJs with an externally controlled heat source (Peltier module and thermistor) and used this calibration to estimate the heat generated by the incident laser light $\left(\Delta \mathrm{T}_{\text {laser }}\right)$ inside the MTJ. A thermal equivalent model [47][48] of an MTJ structure helped to calculate the power required to generate this particular temperature's increase ( $\left.\mathrm{q}_{\text {heat }}\right)$. We retrieved an effective absorption coefficient by comparing this power to the incident laser power.

At first, we calibrated the MTJ thermal response with an external thermo-electric controller (TEC) by measuring the resistance of MTJ $\left(\mathrm{R}_{\mathrm{MTJ}}\right.$ ) at various temperatures (Figure 4(b)). Using linear regressions, we extracted a temperature coefficient of resistance $\left(\alpha_{\mathrm{AP} / \mathrm{P}}\right)$ [49] with temperature's variation for both MTJ states. The temperature difference during the experiment can be calculated from the change in the resistance, described by:

$$
T_{1}^{A P / P}-T_{2}^{A P / P}=\frac{R_{M T J}^{A P / P}\left(T_{1}\right)-R_{M T J}^{A P / P}\left(T_{2}\right)}{\alpha_{A P / P}}
$$

The laser incident on the MTJ can have two thermal effects. Firstly, the absorption of the laser by the MTJ raises the temperature of the pillar. Secondly, the vertical alignment of the laser and the progressively occurring light absorption through the MTJ (Beer-lambert's law) lead to a thermal gradient across the device. This thermal gradient generates a thermal voltage $\left(\mathrm{V}_{\text {th }}\right)$ in an MTJ structure known as the Seebeck effect [50][51], and the thermally influenced resistivity of the tunnel junction has to be considered during the measurements. To estimate and remove the latter effect, we measured the voltage across the MTJ against two symmetrical current source value (i.e. $+\mathrm{I}_{\mathrm{dc}},-\mathrm{I}_{\mathrm{dc}}$ ). Since the thermal gradient from the laser generates the thermal voltage (Seebeck effect), it is independent of the current source direction. This gave us two relations:

$$
\begin{aligned}
& V_{M T J}\left(+I_{d c}\right)=+I_{d c} R_{M T J}+V_{t h} \\
& V_{M T J}\left(-I_{d c}\right)=-I_{d c} R_{M T J}+V_{t h}
\end{aligned}
$$

Combining these two equations (11) isolates the MTJ's resistance:

$$
R_{M T J}=\frac{V_{M T J}\left(+I_{d c}\right)-V_{M T J}\left(-I_{d c}\right)}{\left(+I_{d c}\right)-\left(-I_{d c}\right)}
$$

The MTJ's resistance depended on the measuring current and showed a small asymmetry between polarities. The average of the two-measured voltage had a nonzero value, even without the presence of the laser, which suggested that the MTJ's resistance as an athermal component likely due to the asymmetry of the pillar stack. 

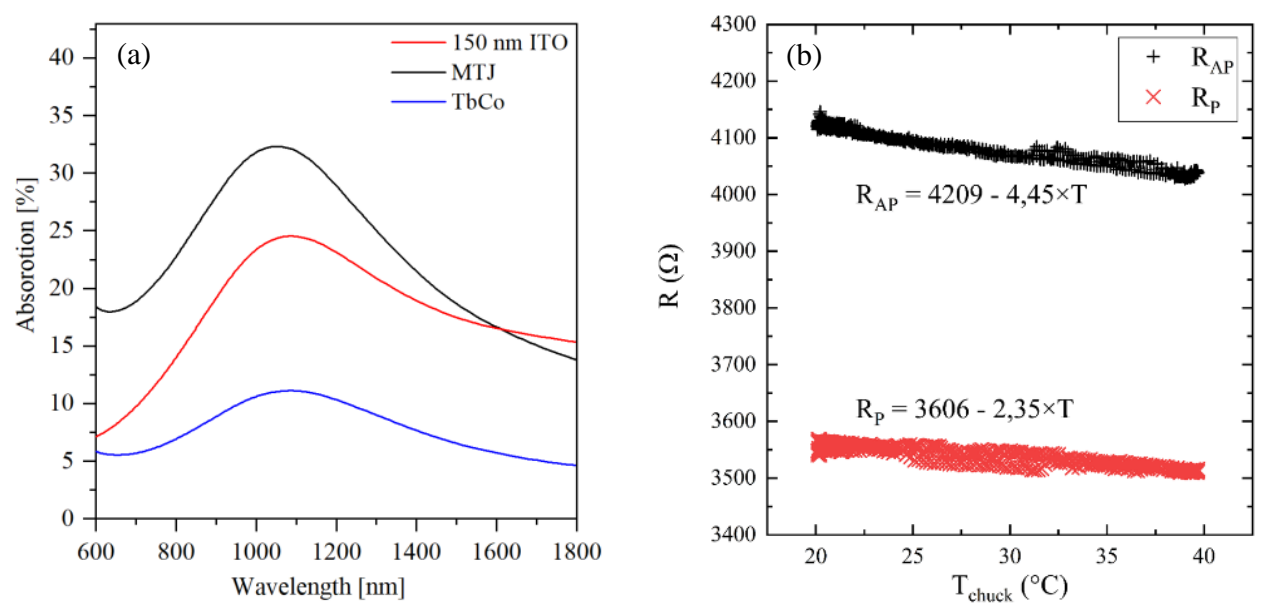

Figure 4 (a) Simulated absorption by $150 \mathrm{~nm}$ of ITO, MTJ layer (Pt/Ta/(Tb/Co)), and in multilayers stack of (Tb/Co) $\times 5$, (b) thermal dependence calibration of an MTJ in both high (AP) and low $(P)$ resistive state.

To characterize the effects of the laser light onto the MTJ, we aligned a single-mode tapered fibre (SMF) with a spot size of $2.5 \pm 0.5 \mu \mathrm{m}$ on top of the MTJ. The alignment was tracked by measuring the resistance feedback continuously from the MTJ. Proper alignment of the continuous wave (CW) laser (at a wavelength of $1550 \mathrm{~nm}$ ) onto the junction was evaluated by the drop of resistance due to the increase of the temperature by the laser light absorption.

We performed a scan of the fibre across the MTJ using piezo controllers with $30 \mu \mathrm{m}$ travel in the XY plane to find a resistance minimum (Figure 5). Using the coefficient of resistance variation with temperature $\alpha_{\mathrm{AP} / \mathrm{P}}$ based on Figure 4 (b), we could calculate the temperature change of the MTJ due to the illumination of the laser (Figure 5). After alignment, we measured the resistance behaviour against the input optical power from our CW laser. This enabled us to estimate the coupling efficiency of incident optical power to absorbed optical power in the MTJ. 

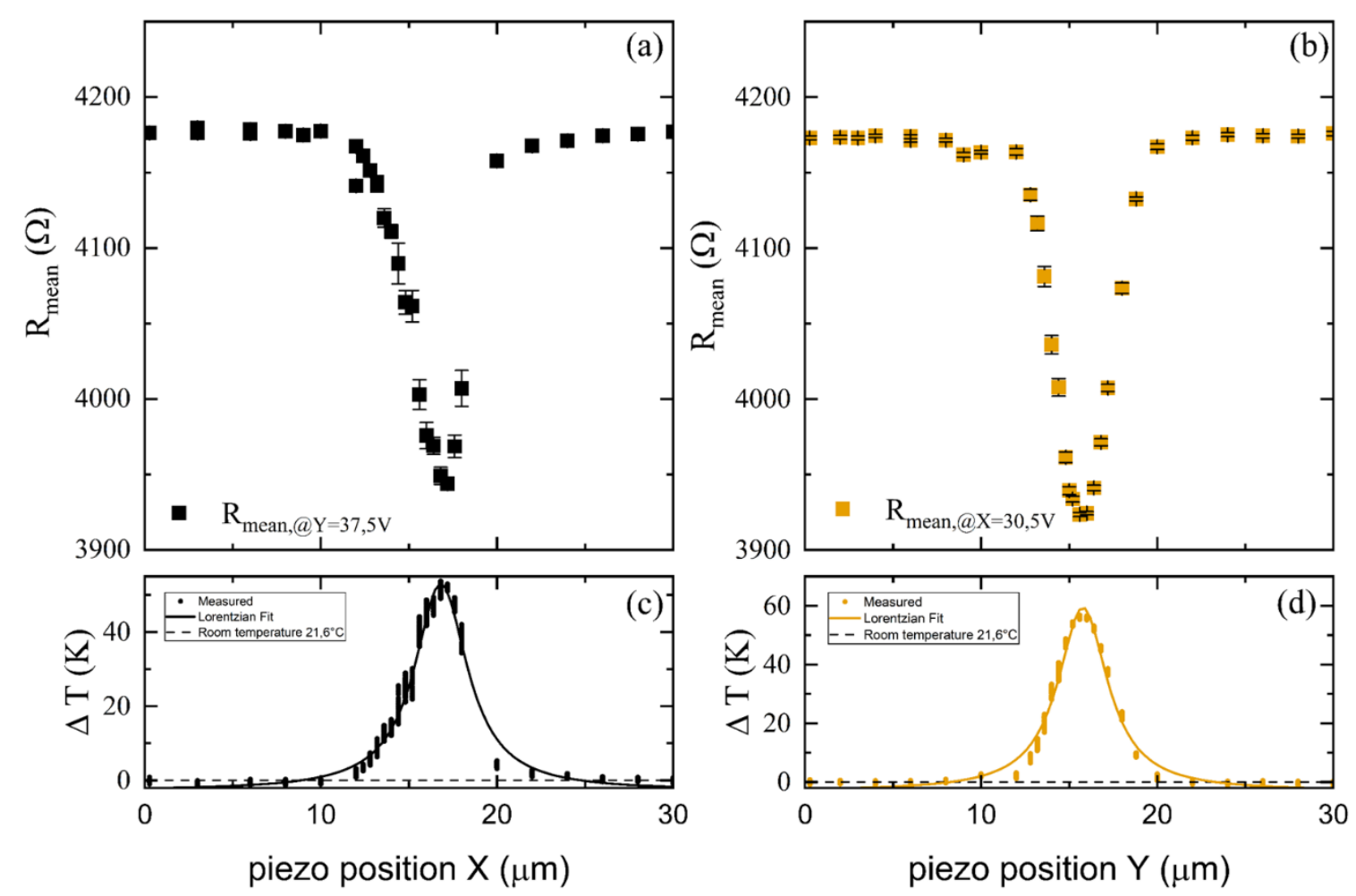

Figure $5(a)$ and (b) The MTJ's resistance and temperature increase $\Delta T$ vs fibre position in X-and $Y$-axis, respectively; (c) and (d) X and $Y$-axis thermal feedback with piezo controller alignment; the full range of piezo controller was $30 \mu \mathrm{m}$ for each axis.

Based on the presented thermal model, we calculated the absorption efficiency for various MTJs. We achieved the best absorption value of about $-8.6 \mathrm{~dB}(14 \%)$ for a $300 \mathrm{~nm}$ diameter MTJ and $-14 \mathrm{~dB}$ $(3.7 \%$ ) for a $200 \mathrm{~nm}$ diameter MTJ. These values include $0.44 \mathrm{~dB}$ of additional losses from a fibre coupler and a polarisation controller located between the laser power meter and the fibre output. Removing these losses give absorption values of $-8.16 \mathrm{~dB}(15 \%)$ and $-13.56 \mathrm{~dB}(4.4 \%)$. Obtained results are consistent with preliminary numerical calculation. Higher convergence for both sets of data should not be required. The numerical model presents the ideal situation excluding the influence of roughness of the layers, incidence angle deviations and structure's shape. Those parameters might bring additional changes in the heat distribution and change the absorption of the junction significantly.

\subsection{Switching mechanism}

An investigation of all-optical single pulse switching of the MTJ electrode was performed with the sample presented in Figure 1(a). For the first experiment, we configured the magnetic top layer parallel with respect to the magnetic bottom layer before the arrival of each laser pulse (Figure 6(a)). After each laser pulse, we measured the magnetic hysteresis as is shown in Figure 6(b) and then extracted the resistance value of the device at $30 \mathrm{mT}$ (Figure 6(c)).

In the second experiment, we applied similar procedures, however, now we configured the magnetic top and bottom layer to be anti-parallel with respect to each other before the arrival of each laser pulse Figure 6(d). The magnetic hysteresis and resistance values after each laser pulse is shown 
in Figure 6(e) and Figure 6(f), respectively. The magnetic hysteresis at pulse 3 and 4 (Figure 6(b), (c)) did not show a reversal, and this was due to mechanical vibrations and drifting of the sample holder resulting in occasional misses of the laser pulse onto the MTJ device target.

Since the applied magnetic field was only strong enough to reverse the magnetization of the bottom layer, we understood a reversal of the magnetic hysteresis behaviour as the switching of the top $\mathrm{Tb} / \mathrm{Co}$ magnetization. Figure 6(b) shows that the hysteresis did not change after the laser pulse excited the MTJ device in the parallel configuration. However, Figure 6(e) shows that the hysteresis did change after the laser pulse excited the MTJ device in the anti-parallel configuration.

The fact that the optical pulse did not affect the magnetic hysteresis of the MTJ device in the parallel configuration (while in the case of the anti-parallel configuration we observed a clear reversal of the magnetic hysteresis) meant that the top magnetic layer must experience a stray field originating from the bottom magnetic layer. It is well known that the magnetization under an ultrashort laser pulse is suppressed at a sub-picosecond timescale [14]. During the recovery period of the magnetization, it became susceptible to small magnetic fields. This high sensitive during the later time could prevent the top layer from switching the magnetization from a parallel to anti-parallel configuration with the bottom layer while without the bottom layer the magnetization of the top layer could switch deterministically under a single laser pulse [8] [10]. To estimate this stray field, we applied a magnetic field at various field strengths during the arrival of the laser pulse. We estimated that an average of 6.3 mT was needed to switch the MTJ device from a parallel configuration to an anti-parallel configuration. 
(a)
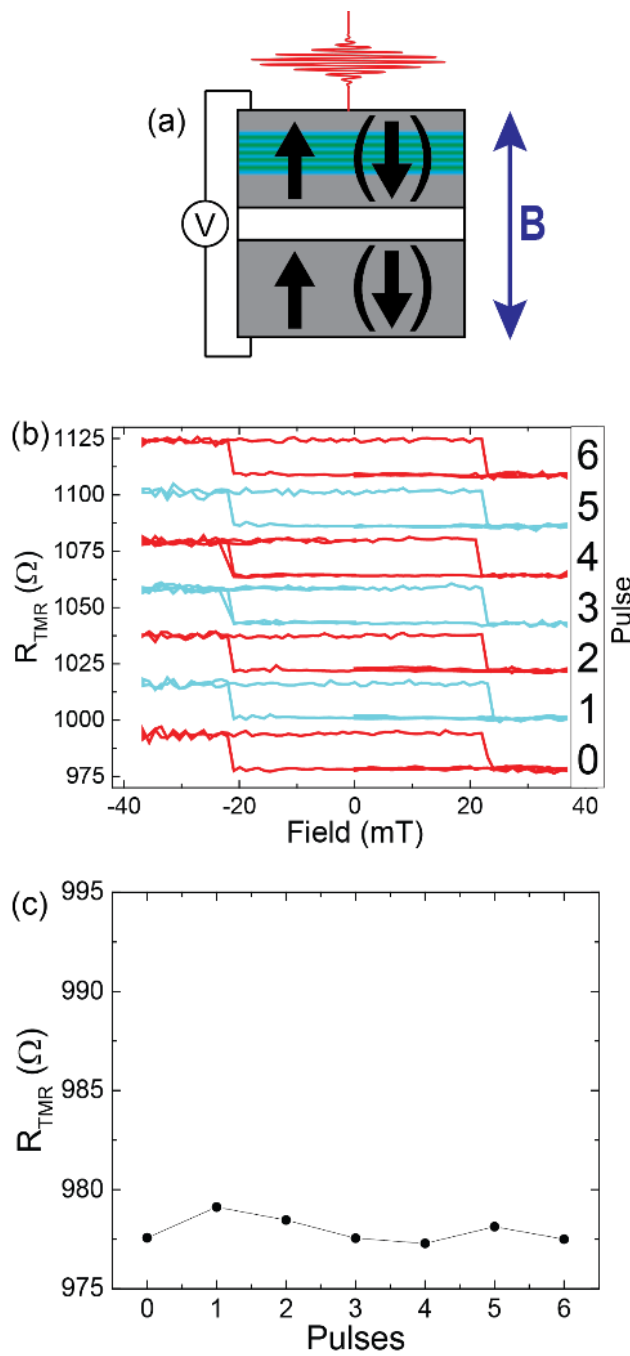

(d)

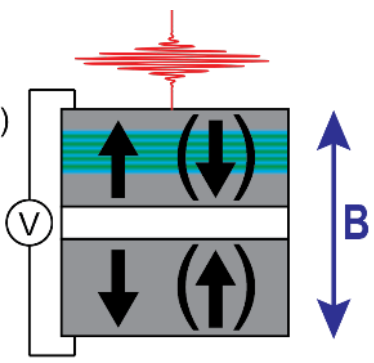

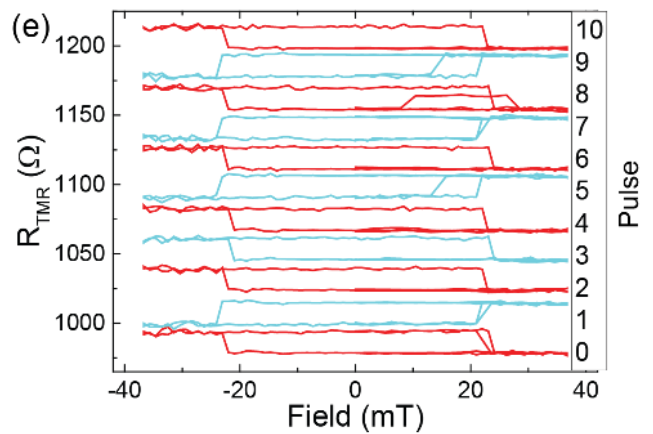

(f)

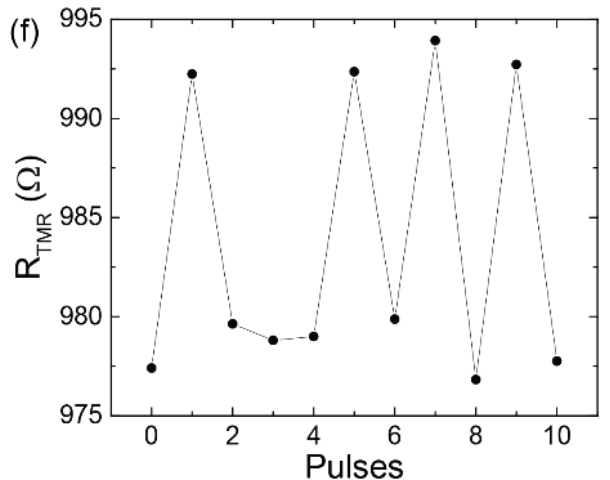

Figure 6. (a) Scheme of the parallel pre-optical-pulse configuration; (b) The magnetic field hysteresis of MTJ device after each laser's pulse with the resistance of the TMR as a function of the applied magnetic field; (c) The resistance at $30 \mathrm{mT}$ is compared after each pulse. The same experiment was performed for $(d)$ the anti-parallel configuration with the respective (e) magnetic field hysteresis and (f) resistance at $30 \mathrm{mT}$.

\subsection{Focusing grating couplers}

We have presented out-of-plane focusing grating couplers (FGCs) as a solution for vertical cointegration in [23]. To enable dense integration, the FGCs were designed for a small footprint, highbandwidths (permitting short optical pulse handling), effective and diffraction-limited focusing and were investigated both in a 1D configuration, as shown schematically in Figure 7 (a), and in a 2D configuration, as shown in Figure 7 (c). Since the binary resistivity state of the MTJs changes, if the absorbed optical energy surpasses a material-specific threshold value, it becomes particularly important to achieve high peak intensities within a small focal area. The 2D FGCs have the additional advantage for full-control of the emitted polarization, given the relative phase control of the two input ports [23]. Design restrictions aroused from the fact that fabrication was done on the standard 220nm-silicon-on-insulator platform available by IMEC [36], defining the layer stack, layer thickness and minimum design feature sizes. 
We experimentally determined the focusing characteristics with a cross-scan procedure using a tapered-and-lensed fibre, evaluating the spatial coupling efficiency to the fibre. The measurements revealed the expected focusing behaviour, indicating a minimum $\mathrm{x}-/ \mathrm{y}-\mathrm{FWHM}$ of $2.3 \mu \mathrm{m}$ and $2.1 \mu \mathrm{m}$ for a well-performing single-silicon-layer 1D FGC and $1.4 \mu \mathrm{m}$ and $2.8 \mu \mathrm{m} \mathrm{x}-/ \mathrm{y}-\mathrm{FWHM}$ values for a well-performing 2D FGC. Given that the experimental analysis relied on the assumption of a Gaussian-like elliptical beam profile, the FGC emission was imaged with a custom-built high-NA microscope and an infrared camera. A recorded image of a double-layer FGC is shown in Figure 7 (b), indicating low side-lobes and a focal spot with a low degree of ellipticity. Figure 7 (d) shows much more pronounced side-lobes in the case of the 2D FGC and a higher degree of ellipticity. While image magnification and resolution, as well as, poor height control of the microscope very likely increased the measured FWHM values, the images revealed the diffraction of a considerable amount of light into the side-lobes, which was intensity lost in the maximum peak.

The experimentally determined focusing characteristics of the designed out-of-plane FGCs revealed the unambiguous focusing behaviour of the emitted beam. Measurement results for the 1D FGC showed relatively uniformly shaped Gaussian beam profiles and FWHM values of the focal spot of a few micrometres. Characterization of the focusing achieved by 2D FGCs also revealed the desired focusing, but measurements implied a more distorted focal spot and intensity diffraction into focal side-lobes. With emission efficiencies not exceeding $-13 \mathrm{~dB}$ for our single-silicon-layer gratings, further optimization of these devices is needed, which should yield higher efficiencies. The doublesilicon-layer gratings showed the potential for improvement with simulated $74 \%(-1.3 \mathrm{~dB})$ directionality.
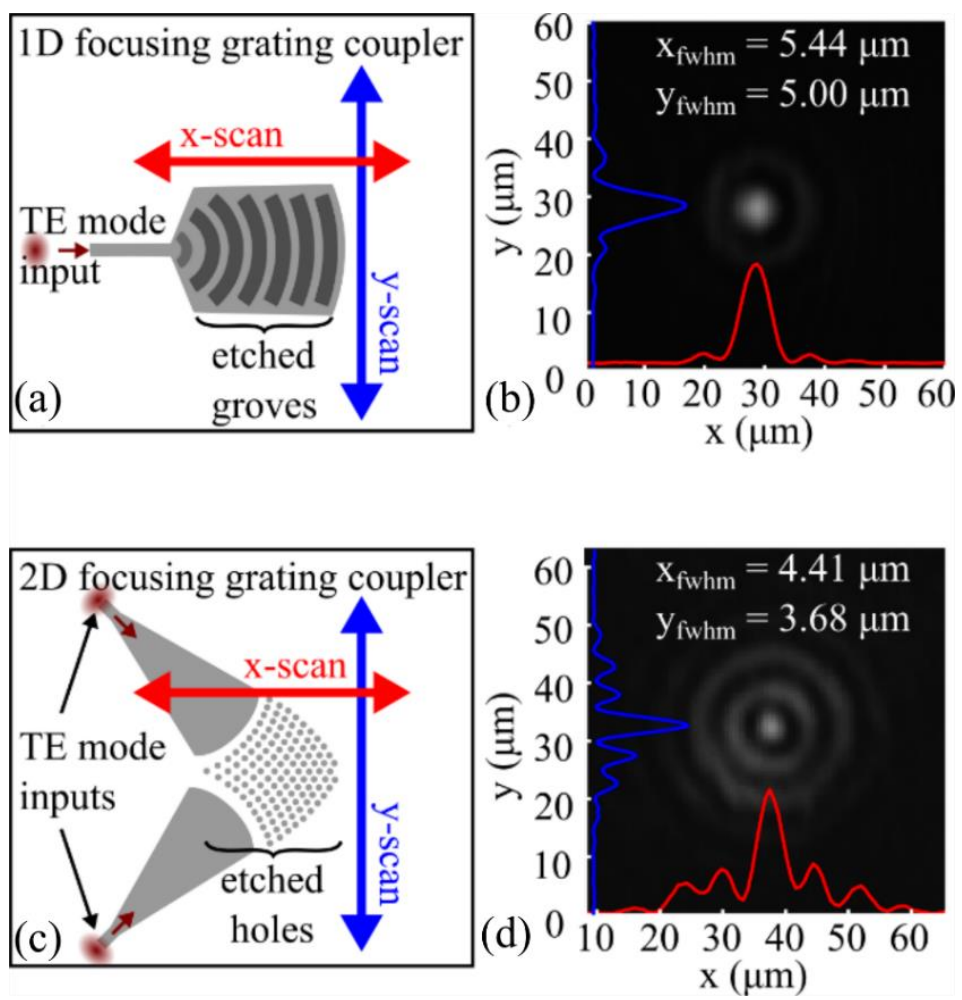

Figure 7 Schematic representation of FGCS and their focal spot imaging by a microscope with IR-camera for 1D FGC (a) and (b), respectively; and (c), (d) for $2 D$ FGC; 


\subsection{Network and output power}

For the all-optical switching event of MTJ cells, sufficient optical pulse energy is required after the photonic distribution network. When composing the photonic network with silicon waveguides, the optical throughput may be limited due to nonlinear loss. At telecom wavelengths, the propagation loss increases with higher optical intensities originating from two-photon absorption (TPA) and consequently resulting in free-carrier absorption (FCA). The saturation of out-coupled power has been shown for CW light and silicon strip waveguides (220nmx450nm) in [52]. Here, we show the power saturation for pulsed light $(5 \mathrm{ps}, 10 \mathrm{GHz}$ repetition rate). The experimental results are illustrated in Figure 8 (a), indicating the limitation of out-coupled optical pulse energy.

From the pulse energy, we calculated the expected optical fluence using focusing grating couplers as designed in [23] with a spot size of $4 \mu \mathrm{m}^{\wedge} 2$. The results are presented in Figure 8 (b). It becomes clear that the fluence is below the requirements for all-optical magnetic reversal. In order to increase this value, we suggest reducing the nonlinear absorption in active structures based on reverse-biased pin-diodes [53][54] or in passive structures based on rib waveguides [52].
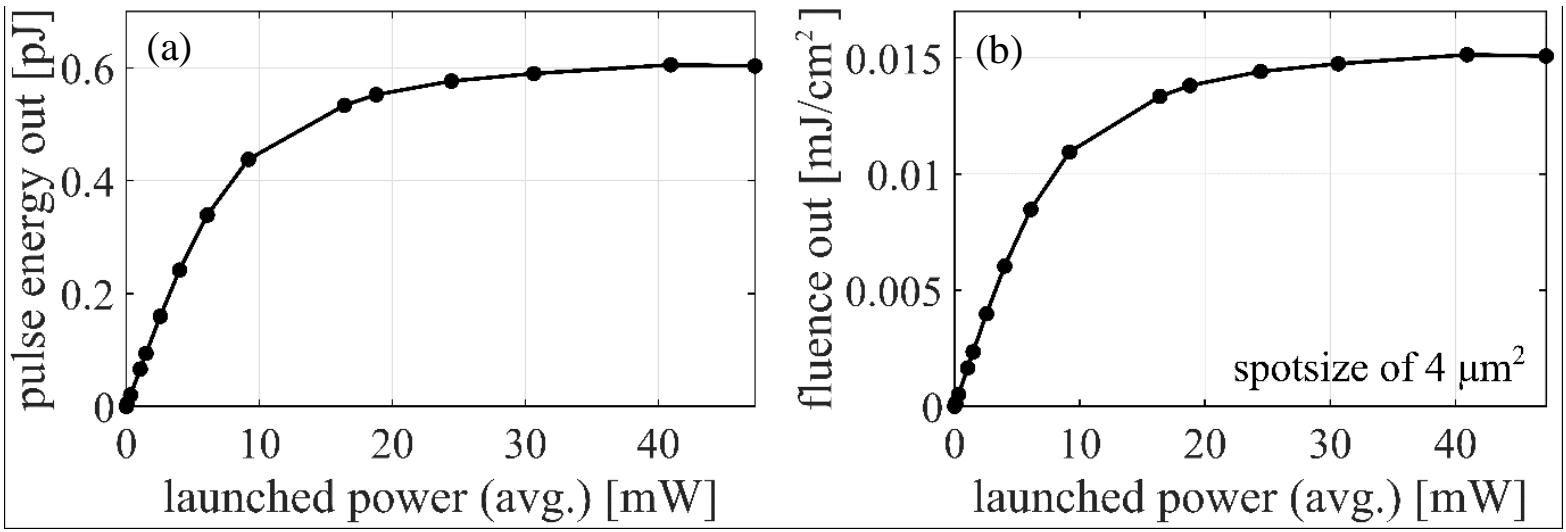

Figure 8 Experimental characterization of nonlinear loss in silicon strip waveguides; (a) Pulse energy after photonic chip vs average power of optical pulse train; (b) Optical fluence after photonic chip vs average power of optical pulse train.

\section{CONCLUSION AND OUTLOOK}

In this work, we showed crucial steps towards spintronic-photonic integration. The ellipsometry investigated the optical properties of the MTJ storage layer stack consisting $\operatorname{Pt}(2 \mathrm{~nm}) / \mathrm{Ta}(2$ $\mathrm{nm}) /[\mathrm{Tbx} / \mathrm{Co} 1.5](13 \mathrm{~nm}) / \mathrm{Si}(5000 \mathrm{~nm})$ before and after annealing. The complex refractive index of the multilayer stack of $(\mathrm{Tb} / \mathrm{Co})_{x 5}$ was determined from a multi-sample analysis of layers with a variety of Tb's thicknesses. Presented results showed higher than previously reported values of the refractive index and an extinction coefficient for Tb, Co or their alloys. This unique behaviour indicated the crystalline-like structure formed by alternating layers of $\mathrm{Tb}$ and $\mathrm{Co}$ in the MTJ. Obtained optical properties were stable for samples annealed at a temperature below $300^{\circ} \mathrm{C}$. Those parameters were implemented into a theoretical FDTD model to calculate absorption in full-sheet films and into the thermal model to estimate effective absorption by the pillar $\left(\mathrm{Pt}_{(2 \mathrm{~nm})} / \mathrm{Ta}_{(2 \mathrm{~nm})} /\left[\mathrm{Tb}_{\mathrm{x}} / \mathrm{Co}_{1.5}\right]_{(13 \mathrm{~nm})}\right)$ in the working device. Results showed high convergence of the results with max $17.5 \%$ and $14 \%$ absorption at $1550 \mathrm{~nm}$ for the 300nm diameter MTJs from simulations and experiments, respectively. 
Single-pulse switching measurements on the MTJ electrode showed that the reversal of the magnetic hysteresis was possible only in the case of anti-parallel configuration. These results suggested that the top magnetic layer became susceptible to small magnetic fields from the bottom layer during short light pulses, and in consequence, prevented switching of the optically switchable layer. We estimated that the strength of a stray field that is needed to switch the MTJ device from a parallel to an anti-parallel configuration is at an average of $6.3 \mathrm{mT}$. This finding shows that all-optical switching is possible regardless of the starting configuration with proper adjustment against the intrinsic stray field. Next crucial steps towards proper integration are to provide an adequate amount of the laser light to the MTJs. We proposed and investigated integrated silicon photonics networks with focusing grating couplers in terms of sufficient out-coupled energy and the spot size. The FGCs offer a small footprint and show the potential for highly efficient coupling. Even though the silicon photonic platform limits the maximum optical output power due to nonlinear losses, we can conclude from the presented experimental data, that it is, in fact, suitable for integrating photonics with electronics for both monolithic and hybrid integration schemes,

However, the footprint mismatch between the photonics and electronics technology remains large. Scaling micrometre sized and diffraction-limited photonic components to nanometre-sized electrical components remains a technological challenge. In addition, a knowledge of all the mechanisms in the AOS structure is crucial to overcome the energy losses that are limiting the performance of the integrated device. Energy improvement can be done in many ways from applying active elements in the network, fabricating antireflection coating on top of the MTJ up to implementing plasmonic structures for enhancing the electromagnetic field in the junction's structures. These developments represent the next step towards the integration of spintronic-photonic systems on the chip-level. Feasible further optimization of the elements opens a new path for faster memory devices with lower energy consumption.

\section{REFERENCES}

[1] Prejbeanu, I. et al., "Thermally assisted MRAM", Journal of Physics: Condensed Matter 19 (16), 1065-1100 (2007)

[2] Hu, J.-M., et al., "High-Density magnetoresistive random access memory operating at the ultralow voltage at room temperature", Nature Communications 2 (1), 553-558 (2011)

[3] Kan, J.J., et al., "A study on practically unlimited endurance of STT-MRAM", IEEE transactions on electron devices64 (9), 3639-3646 (2017)

[4] Chen, J., et al., "All-Optical Switching of Magnetic Tunnel Junctions with Single Subpicosecond Laser Pulses," Physical Review Applied 7, 021001, 2-7 (2017), doi: https://doi.org/10.1103/PhysRevApplied.7.021001

[5] Kimel, A. V., Li, M., "Writing magnetic memory with ultrashort light pulses," Nature Reviews Materials 4, 189200 (2019), doi: https://doi.org/10.1038/s41578-019-0086-3

[6] Stanciu, C.D., et al., "All-optical magnetic recording with circularly polarised light", Physical Review Letters 99 (4), 1-4 (2007)

[7] Lalieu, M. L. M., et al., "Integrating all-optical switching with spintronics", Nature Communications 10 (1), 1-6 (2019)

[8] Avilés-Félix, L., et al." Single-shot all-optical switching of magnetization in Tb/Co multilayer-based electrodes", Scientific Reports 10, 5211 (2020). https://doi.org/10.1038/s41598-020-62104-w

[9] Ciuciulkaite, A. et al." Design of Amorphous TbxCo100-x Alloys for All-Optical Magnetization Switching", Applied Physics (2020)

[10]Avilés-Félix, L. et al., "Integration of Tb/Co multilayers within optically switchable perpendicular magnetic tunnel junctions", AIP Advances 9, 125328 (2019); 
[11] Olivier, A., et al., "Indium Tin Oxide optical access for magnetic tunnel junctions in hybrid spintronic-photonic circuits", Nanotechnology 31 (2020)

[12] "Spice project - developing future memory", Available online: http://spice-fetopen.eu/

[13]Davies, C.S., et al., "Pathways for Single-Shot All-Optical Switching of Magnetization in Ferrimagnets", Physical Review Applied 13, 024064 (2020)

[14]Beaurepaire, E., et al., "Ultrafast spin dynamics in ferromagnetic nickel," Physical Review Letters 76, (22), 4250-4253 (1996)

[15]Hilfiker, J.M. et al., " Survey of methods to characterize thin absorbing films with Spectroscopic Ellipsometry", Thin Solid Films 516, 7979-7989 (2008)

[16]Losurdo, M., Hingerl K., “"'Ellipsometry at the nanoscale”, Springer Heidelberg New York Dordrecht London, $1-30$ (2013)

[17]Palik, E.D., Lynch, D.W., Hunter, W.R," Handbook of Optical Constants of Solids I; - An Introduction to the Data for Several Metals", Academic Press, 341-419 (1997)

[18]Palik, E.D., Lynch, D.W., Hunter, W.R," Handbook of Optical Constants of Solids I; Comments on the Optical Constants of Metals and an Introduction to the Data for Several Metals ”, Academic Press, 275-367 (1997)

[19]Iakovlev, S., et al., "Rare-earth ions doping effects on the optical properties of sol-gel fabricated PbTiO3 thin films", Thin Solid Films 446 (1), 50-53 (2004)

[20] Johnson, P. B., Christy, R. W., "Optical constants of transition metals: Ti, V, Cr, Mn, Fe, Co, Ni, and Pd”, Physical Review B 9, 5056-5070 (1974)

[21]Palik, E.D., Edwards D. F.," Handbook of Optical Constants of Solids I; - - Silicon (Si)*”, Academic Press, 547569 (1997)

[22]Nanophotonic FDTD Simulation Software - Lumerical FDTD Solutions, (2018). https://www.lumerical.com/products/fdtd-solutions/.

[23] H. Becker, et al., "Out-of-plane focusing grating couplers for silicon photonics integration with optical MRAM technology,” IEEE Journal Of Selected Topics In Quantum Electronics 26, (2019).

[24] Surabhi, S., et al., "Precise Determination of the Temperature Gradients in Laser-irradiated Ultrathin Magnetic Layers for the Analysis of Thermal Spin Current", Scientific Reports 8, 11337 (2018); https://doi.org/10.1038/s41598-018-29702-1

[25] Stephens, R. E., Malitson, I. H., "Index of refraction of magnesium oxide", Journal of Research of the National Bureau of Standards 49, 249-252 (1952)

[26] Nanophotonic FDTD Simulation Software - Lumerical FDTD Solutions, "Stack optical solver overview", (2018),

[27]Nanophotonic FDTD Simulation Software - Lumerical FDTD Solutions, "Calculating absorbed optical power Higher accuracy", available online; (2018)

[28]Le Guyader, L., et al., "Nanoscale sub-100 picosecond all-optical magnetization switching in GdFeCo microstructures," Nature Communications 6 (2015); doi: 10.1038/ncomms6839

[29] Savoini, M., et al., "Highly efficient all-optical switching of magnetization in GdFeCo microstructures by interference-enhanced absorption of light," Physical Review B 86 (2012); doi: 10.1103/PhysRevB.86.140404.

[30]WebElements, "The periodic table", Available Online (25th August 2020), https://www.webelements.com

[31]Pakanen, J.," Conduction of heat through slabs and walls", VTT Publications 169 (2009),) available online (8th August 2020), www.vttresearch.com/sites/default/files/pdf/publications/2009/P162.pdf

[32] Nave, R., Department of Physics and Astronomy, Georgia State University, Online tutorials from physics (2016), available online (30th July 2020), hyperphysics.phy-astr.gsu.edu/hbase/thermo/heatra.html\#c1

[33] Taler, J., Duda, P., "Solving direct and inverse heat conduction problems", Springer Science \& Business Media, 2010

[34]Taillaert, D. F., et al., "Grating couplers for coupling between optical fibres and nanophotonic waveguides", Japanese Journal of Applied Physics, Part 1: Regular Papers and Short Notes and Review Papers 45 (8), 60716077 (2006)

[35] Michaels, A., Yablonovitch, E., "Inverse design of near-unity efficiency perfectly vertical grating couplers", Optics Express 26 (4), 4766-4779 (2018)

[36] Silicon photonic ICs for prototyping, Available Online (10th June 2020) https://www.imec-int.com/en/siliconphotonic-ICs-prototyping

[37]Palik, E. D., Ward, L., „Handbook of Optical Constants of Solids III - Optical Constants of Eight Rare Earth Elements: (Ce), (Sm), (Gd), (Tb), (Dy), (Er), (Tm), and (Yb)”, Academic Press, 287 - 340 (1997) 
[38]Palik, E. D., Ward, L., „Handbook of Optical Constants of Solids II - Cobalt (Co)”, Academic Press, 435 - 448 (1997)

[39]Pavlov, V. et al., "Ellipsometric and magneto-optical study of nanosized ferromagnetic metal-dielectric structures [Co/TiO2]n/Si", Thin Solid Films 619 (2016)

[40]Farmakidis, N., et al., "Plasmonic nanogap enhanced phase-change devices with dual electrical-optical functionality", Science Advances 29 (2019)

[41]Hoffmann, M. A., et al., "Spectroscopic ellipsometry and magneto-optical Kerr effect spectroscopy study of thermally treated Co60Fe20B20 thin films", Journal of Physics: Condensed Matter 32 (2020),

[42] Liu, Y., et al., "Effects of annealing temperature, thickness and substrates on optical properties of m-plane $\mathrm{ZnO}$ films studied by photoluminescence and temperature dependent ellipsometry", Journal of Alloys and Compounds [In Press] (2020)

[43] Alfonso, J.E., et al., "Annealing Effect on the Structural and Optical Properties of Sputter-Grown Bismuth Titanium Oxide Thin Films", Materials 7, 3427-3434 (2014)

[44]Mahbubur Rahman, M., el. al., " Investigation of the post-annealing electromagnetic response of $\mathrm{Cu}-\mathrm{Co}$ oxide coatings via optical measurement and computational modelling", RSC Advances 27 (2017)

[45] Hojabri, A., et al., "Annealing temperature effect on the properties of untreated and treated copper films with oxygen plasma", Journal of Theoretical and Applied Physics 8, 132 (2014). https://doi.org/10.1007/s40094-0140132-x

[46] Manouchehri, I., et al., "Investigation of annealing effects on optical properties of Ti thin films deposited by RF magnetron sputtering", Optik 127 (13), 5383-5389 (2016)

[47]Lecture notes for Aerospace Engineering at Sapienza Università di Roma, "Chapter 3: Thermal-electrical analogy: thermal network", available online (4th http://www.ingaero.uniroma1.it/attachments/2176_Cap_3\%20Thermal-electrical\%20analogy.pdf;

[48]Cheever, E. Department of Engineering, Swarthmore College, Complementary notes for the course - Linear Physical Systems Analysis; "Mathematical Models of Thermal Systems", available online (4th August 2020): https://lpsa.swarthmore.edu/Systems/Thermal/SysThermalModel.html;

[49] Yang, H. F. et al., "Electrical measurement of absolute temperature and temperature transients in a buried nanostructure under ultrafast optical heating," Applied Physics Letters, vol. 110, no. 23, p. 232403, Jun. 2017, doi: $10.1063 / 1.4985434$.

[50]Walter, M. et al., "Seebeck effect in magnetic tunnel junctions," Nature Materials, vol. 10, no. 10, pp. 742-746, Oct. 2011, doi: 10.1038/nmat3076

[51]Liebing, N., et al., "Tunneling Magnetothermopower in Magnetic Tunnel Junction Nanopillars," Physical Review Letters, vol. 107, no. 17, Oct. 2011, doi: 10.1103/PhysRevLett.107.177201.

[52] Krückel, C. J., et al., "Impact of optical free-carrier generation on the performance of SOI phase shifters", Conference on Lasers and Electro-Optics, OSA Technical Digest (Optical Society of America, 2020), paper SM2J.3

[53] Gajda, A. et al." Highly efficient CW parametric conversion at $1550 \mathrm{~nm}$ in SOI waveguides by reverse biased pi-n junction," Optic Express 20, 13100-13107 (2012)

[54]Turner-Foster, A. et al.," Ultrashort free-carrier lifetime in low-loss silicon nanowaveguides," Opt. Express 18, 3582-3591 (2010) 